\title{
Performance Analysis of Distributed Real Time Optimization Technique for Optimal Power Flow Problems
}

\author{
Shweta Singh, Rajnish Bhasker
}

\begin{abstract}
For conventional electric power system, penetration of high level of distributed generation is new challenge. Distributed energy resources produces on-site electricity, by which it reduces the requirement of new transmission line set up and also provides reduction of line losses. In earlier days, centralized optimization approaches have been the primary way of optimization of power system. In centralized approach of optimization, optimal power flow operation is performed by collecting information at central controller. But because of increase of size of power system and enhancement of distributed resources from demand side various problem arises in performing computation and control in centralized approach. Optimal operation of power flows are utilized in planning and scheduling of generation of power. Most of the non-conventional resources are located at the distribution grid. So to handle the uncertainness associated in renewable energy resources, load demands etc. there is a need of development of distributed algorithm for AC-optimization problems. This paper realizes in real time optimization and to track the time varying $O P F$ problem and for numerical optimization method different quasi-Newton methods are used. For time varying loads there is a requirement of an algorithm technique that can track variant load on faster time scale. So, to address these challenges various approaches or techniques are presented.
\end{abstract}

Key words - power optimization, distributed optimization technique, real time optimization, electric power grid

\section{INTRODUCTION}

As we know that the conventional power grid consist of generating stations, transmission lines which provides transfer of power from power generating station to distribution grid, and distribution grid provides generated power to consumers at load side.[1] The conventional grid transfers power from generating side to load side i.e. unidirectional. Conventional grid provides conversion of only one third of fuel input into electric power because of heat losses at generating station and transmission losses at transmission line. So to reduce these losses and improve efficiency of power grid renewable energy resources are located at distribution side. Renewable energy resources are introduced to provide reduction of carbon emission, improvement of stability and efficiency of arrangement.[2] In earlier days, power system research was mainly done on the central part of grid, can say that started from generating side through transmitting part and then to substations. On core network of power system optimization and control was done by centralized approaches like an ISO.[3], [4], [4]-[9] But now the focus is shifted towards the distribution side

Revised Manuscript Received on 14 August, 2019.

Shweta Singh, Department of Electrical Engineering, UNSIET,VBS Purvanchal University, Jaunpur, Uttar Pradesh, India.

RajnishBhasker, Department of Electrical Engineering, UNSIET, VBS Purvanchal University, Jaunpur, Uttar Pradesh, India. due to integration of renewable energy resources, ability of self healing and demand response and this new structure is called as smart grid. The most important problems in smart grid are optimal power flow. The aim of OPF is optimization of generation cost which is subjected to constraints of demand and network.[6], [10]-[17]. For solving the OPF heuristic approaches were employed generally but by this approach incurring of optimal solution are not guaranteed. Assumptions like lossless parameters provides easy calculation, make the equation linear and this obtained equation is called as DC-OPF.[18] This DC-OPF will not provide accurate solution under all circumstances problems of optimization and control are formulated by using network parameters like impedances of line, parameters of generators, network topology, load parameters, flow and output limits. So in centralized computation ISO (Independent System Operator) collects all required parameters and perform computation centrally. [19] In distributed optimization algorithm instead of collecting parameters of all problems and performing a calculation centrally, it obtain

Problem parameters via communication with limited neighbours set and perform computation by many agents. [20]-[22]

There are various advantages of using distributed algorithms over centralized algorithms some of these are: (a) cyber security improvement and reduction of cost of communication infrastructure required, (b) advantageous in terms of data privacy, constraints, costing function and measurements, (c) distributed algorithms are preferable to use instead of using central optimization controller because of solution speed, can address maximum problem size.[23][27] By decentralized algorithm technique, failure of communication between regions can be handled easily than in a centralized algorithm technique because processor in each region can attend local needs.[28]-[30] It is important to solve optimal power flow efficiently because of increase of penetration of renewable energy resources. So effective solving of optimal power flow can be used in real time application. [31][32]

Organisation of analysis is given as: In first section introduction is given. In section II overview of distributed model of power system is provided. In section III formulation of optimal power flow problem and the important background is provided. In section IV summary of various distributed optimization techniques are given. 
Section $\mathrm{V}$ gives the overview of real time optimization of power flow equation

\section{OVERVIEW OF DISTRIBUTED MODEL OF POWER SYSTEM AND OPTIMAL POWER FLOW PROBLEM}

\section{A. Distributed model of power system: -}

In this section we will summarize the equation of power flow with some approximations and assumptions that are relevant with distributed technique of optimization.

First we consider a distributed network of power system with n-no. of busses. Each bus is associated with voltage phases, active as well as reactive power injections.

$$
\text { Let, } \quad V_{i}=\left|V_{i}\right| \angle \theta_{i}
$$

Where $V_{i}$ is the voltage of $i_{t h}$ bus, which means vector voltage of bus.

$$
\begin{aligned}
& V=\left[V_{1} V_{2} V_{3} \ldots \ldots \ldots V_{n}\right]^{T} \in C^{n} \\
& P_{i}+j Q_{i}
\end{aligned}
$$

$P_{i}$ represents active power injected at the $i_{t h}$ bus and

$Q_{i}$ represents the injection of reactive power at the $i_{t h}$ bus.

And vector representation of given active and reactive power are represented as,

$$
\begin{aligned}
& P=\left[P_{1} P_{2} P_{3} \ldots \ldots \ldots P_{n}\right]^{T} \\
& Q=\left[Q_{1} Q_{2} Q_{3} \ldots \ldots \ldots Q_{n}\right]^{T}
\end{aligned}
$$

Admittance of line (i, k) is represented as

$$
y_{i k}=g_{i k}-\mathrm{j} b_{i k}
$$

Where, $g_{i k}$ is conductance of line (i, k)

And $b_{i k}$ is susceptance of line $(\mathrm{i}, \mathrm{k})$

Let shunt admittance of the $i_{t h}$ bus is $y_{i i}=\mathrm{j} b_{i i}$

The active power flowing via transmission line ( $\mathrm{i}, \mathrm{k}$ ) can be represented as,

$$
\begin{gathered}
P_{i k}=\left|V_{i}\right|^{2} g_{i k}\left|V_{i}\right|^{2} g_{i k} \\
\left|V_{i}\right|\left|V_{k}\right|\left[b_{i k} \sin \left(\theta_{i k}\right)-g_{i k} \cos \left(\theta_{i k a}\right)\right]
\end{gathered}
$$

The reactive power flowing via transmission line $(i$, k) can be represented as,

$$
\begin{gathered}
Q_{i k}=\left|V_{i}\right|^{2} b_{i k}-\left|V_{i}\right|\left|V_{k}\right|\left[g_{i k} \sin \left(\theta_{i k}\right)+b_{i k} \cos \left(\theta_{i k}\right)\right] \\
\left|V_{i}\right|^{2} b_{i k}-\left|V_{i}\right|\left|V_{k}\right|\left[g_{i k} \sin \left(\theta_{i k}\right)+b_{i k} \cos \left(\theta_{i k}\right)\right]
\end{gathered}
$$

Where, $\theta_{i k}=\theta_{i}-\theta_{k}$

\section{OVERVIEW OF OPF AND ITS FORMULATION}

OPF was mainly used for planning purpose in traditional power grid like it was used to find the state of system .But in smart grid, OPF techniques are used for handling various uncertainties involved in renewable energy resources and load demands.[33]

A. Problem formulation of OPF: - -
Performance of system are optimized by OPF problem on the basis of specified objective function.

Given objective function $\mathrm{F}$ are represented on the basis of generation cost.

i.e. $F=\sum i \in \varsigma c_{2, i} P_{G i}^{2}+c_{1, i} P_{G i}+c_{0, i}$

Where, $c_{2, i} \geq 0, c_{2, i} \geq 0$,

$c_{1, i}$ and $c_{0, i}$ are scalar coefficients of generator at $i_{t h}$ bus.

$P_{G i}=$ power generated at the $i_{t h}$ bus.

$\varsigma=$ set of generator buses.

Losses are represented as, $\mathrm{F}=\sum i \in N P_{G i}$

Proximity to desired voltage profile are represented as, F

$=\sum i \in N\left(\left|V_{i}\right|^{2}-\left|V_{i}^{*}\right|^{2}\right)^{2}$

$\left|V_{i}^{*}\right|$ is desired voltage profile.

Some constraints are subjected for minimization of generation cost, and for minimization of optimization problem other subjected constraints are physical constraints like limits of power and bus voltage etc.

The OPF problem given here is,

Min F

Subjected to $\left|V_{i}\right|=V_{i}^{r e f}\left|V_{i}\right|=V_{i}^{r e f} \forall i \in N$

$P_{i}^{\min } \leq P_{i} \leq P_{i}^{\max } \forall i \in N$

$Q_{i}^{\min } \leq Q_{i} \leq Q_{i}^{\max } \forall i \in N$

$\left(V_{i}^{\min }\right)^{2} \leq\left|V_{i}\right|^{2} \leq\left(V_{i}^{\max }\right)^{2} \forall i \in N$

$f_{i k}\left(V_{i,} V_{k}\right) \leq I_{i, k}^{\max } \forall(i, k) \in L$

Where, $f_{i k}\left(V_{i}, V_{k}\right)$ denotes the appropriate flow function for line $(\mathrm{i}, \mathrm{k}) \in \mathrm{L}$.

Solving of problem by centralized approach is not practical if size of system increases i.e. the number of buses increases. So to tackle this problem distributed optimization techniques are used.

\section{DISTRIBUTED OPTIMIZATION TECHNIQUE}

Distributed optimization techniques are employed in problems due to limitations of computation and memory limits. It is difficult to summarize whole optimization problem centrally because problems of different grids are known by different subsystems.

In given segment brief reviews are presented on how optimisation techniques are utilized in a distributed way. Various distributed optimization techniques are (1) Primal decomposition method, (2) Dual decomposition method, (3) ATC, (4) APP, (5) OCD, (6) CI.

\section{A. Primal decomposition:-}

The idea of decomposition comes up primarily for solving very large problems which were difficult to solve by using standard optimization techniques.

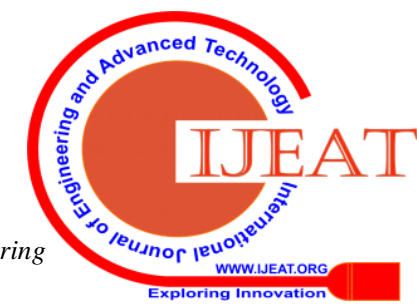


Primal decomposition method is simplest decomposition method. In this subsystem coordinates choose some primal variables. Consider an unconstrained minimization problem,

$$
\text { Minimize } \mathrm{F}(\mathrm{x})=F_{1}\left(x_{1}, y\right)+F_{2}\left(x_{2}, y\right)
$$

Where variable denoted by $\mathrm{x}$ is $\left(x_{1}, x_{2}, y\right)$.

Let $\phi_{1}(y)$ denotes the optimum point of the problem given above.

In similar way, let $\phi_{2}(y)$ denotes the optimum point of problem.

The problem given in equation can be rewritten as,

$$
\text { Minimize y of } \phi_{1}(y)+\phi_{2}(y)
$$

Addition of the optimal values of sub problems is master problems.

So primal method of decomposition is the basic decomposition technique as in this primal variables of master algorithm are gets manipulated. Very simple decomposition algorithm can be obtained on solving master problem by using sub gradient method.

\section{B. Dual decomposition method:-}

This can be expressed as follows:-

$$
\text { Minimize }_{\text {s.t. } y_{1}=y_{2}} F(x)=F_{1}\left(x_{1}, y_{1}\right)+F_{2}\left(x_{2}, y_{2}\right) \text {. }
$$

As the optimization problem of the Lagrangian functions which have a separable structure can be solved by using dual decomposition techniques.

The Lagrangian is expressed as,

$\alpha^{k}$

An iterative method used by dual decomposition called as "dual ascent".

$$
\begin{aligned}
& x_{i}^{k+1}=\arg \min _{x_{i}} L_{i}\left(x_{i}, y^{k}\right) \\
& y^{k+1}=y^{k}+\alpha^{k}\left(\sum_{i=1}^{N}\left(A_{i} x_{i}^{k+1}\right)-b\right)
\end{aligned}
$$

Where, $\alpha^{k}$ is the step size of specific iteration.

C. Alternating Direction Method of Multipliers: - ADMM algorithm was obtained after amalgamation of two earlier proposed algorithms. These two proposed algorithm are based on algorithm of dual ascent and multipliers method which is used for the solution of the augmented Lagrangian problems in distributed way.[34], [35]

The form of optimization problem on which ADMM is applicable is

$$
\begin{aligned}
& \min _{x, y} f(x)+g(y) \\
& \text { s.t.Px+Qy = B }
\end{aligned}
$$

Where, $\mathrm{P}$ and $\mathrm{Q}$ are specified matrices.

$\mathrm{X}$ and $\mathrm{y}$ are decision variables.

$B$ is specified vector.

$\mathrm{f}(\mathrm{x})$ and $\mathrm{g}(\mathrm{y})$ are specified functions.

On the basis of augmented Lagrangian the ADMM are represented as:

$$
L=f(x)+g(y)+s^{T}(P x+Q y-c)+\rho / 2\|P x+Q y-c\|_{2}^{2} .
$$

D. Analytical target cascading:-

ATC is based on iteration and hierarchical technique of solution of an optimal problem. The sub problems obtained by splitting of optimization problem in ATC are related with each other by a tree structure. Convergence of algorithm is guaranteed if all the sub problems are convex.

\section{E. Auxiliary problem principle:-}

as similar to techniques used above the optimization problem of APP technique also get decomposes into subproblems. To ensure consistency between the sub-problems on augmented Lagrangian approach is used. In this also if sub problems are convex then convergence is guaranteed.

F. Optimality condition decomposition: -

In this technique, in its sub-problem it can vary its associated value only. A central coordinator is not needed by OCD techniques. In this technique when the coupling between sub-problems is weak then only an effective condition for convergence holds

G. Consensus + Innovation: -

As in above technique each variable gets associated to specific problems but in given method all variables in given problem can vary by using an iterative algorithm. Except this above conditions all other condition of above two methods are similar. $\mathrm{C}+\mathrm{I}$ technique carry out computation in distributed way, it does not require any central coordinator.

Methods used above have some specific tunable parameters which can be tuned as per the requirement to enhance operation of the arrangement. The step size parameters $\rho$ are the parameters on which performance of ADMM-method depends. Performance of various methods or techniques are basically problem dependent. As different optimization techniques have different speed of convergence, level of accuracy, efficiency of performance, and speed of computation, so approach of selection depends on the requirement of user, and kind of application. No any single technique can perform satisfactory operation in all situations.

\section{REAL-TIME OPTIMIZATION AND CONTROL\& RESULTS}

For future applications of power system, real time optimization may require in large power system network with distributed renewable energy resources. For time varying optimization problem, various online algorithms have been developed. In offline algorithms, in each iteration one cannot apply final solution to the power system network until the convergence of which occurs to equilibrium.

The two main advantages of online closed loop system with optimization algorithm are:- it tracks the changing network condition naturally therefore this algorithm provides robustness to disturbances and uncertainties of fluctuations in loads. For future application in distributed energy of large network the centralized approaches will not be convenient because of cost of collection and communication of required state is high and because of the need of protection of personal messages or information's. So for such requirements, the only viable solution is online optimization or real time distribution 


\section{PERFORMANCE ANALYSIS OF DISTRIBUTED REAL TIME OPTIMIZATION TECHNIQUE FOR OPTIMAL POWER FLOW PROBLEMS}

As for real-world problems, mathematical formulations are derived on the basis of some assumptions and under these assumptions also it is not easy to get dissociated result from power system of large network. As we know that output in renewable energy resources are not certain due to which it is difficult to forecast the output accurately and difference occurs in between actual output and forecasted output. And hence safety problems cannot be tackled properly because of violation in constraints due to uncertainty in output. Therefore for real time operation deterministic optimal power flow techniques are not appropriate. Since, conventional optimal power flow techniques are suitable only for slow time scale applications. For numerical technique to get optimum point different quasi-Newton methods are used. Time variant optimization algorithm satisfies operational constraints on the basis of specific algorithm and also satisfies the power flow equation by design. Online algorithm of optimal power flow problem update the control value $u(t)$ for every interval and this update is done by using controller and then controller applies this control variable to the grid. Power flow equation $\mathrm{F}(\mathrm{x}, \mathrm{u}(\mathrm{t}))=0$ is solved by grid to find out the state variable and then use this state variable to find the next control variable $u(t+1)$ for next time period. So can say that time varying optimisation method calculates a control value $u(t)$ in each iteration and then apply this $u(t)$ to the grid and then grid solve the power flow equation at real time scale and hence calculate the state $\mathrm{x}(\mathrm{t})$. In time varying optimisation algorithm based on quasi Newton methods, for tracking of optimum point, we find the constraints and values of decision variables and then to calculate a minute correction we utilizes them as the initial point.

\section{CONCLUSION}

As the renewable energy resources brings new challenges in operation of power system network. Due to fluctuations in renewable energy resources the network operator has to update the operations in fast way. So this can be performed by real-time optimization. This paper realizes the challenges in optimal power flow and real time optimization and hence to address these challenges various approaches or techniques are presented. In this paper various topics are reviewed in sequential way like OPF, distributed optimization and then real time OPF and the main purpose was to identify recent promising method used for optimization. To address challenges like less computation time, good accuracy and convergence, tracking of the changing network condition naturally various methods are explored and hence it can be concluded that there is not sufficient research on online optimization. So for that new solution techniques are required.

\section{REFERENCES}

1. F. F. Wu, K. Moslehi, and A. Bose, "Power System Control Centers: Past, Present, and Future," Proc. IEEE, vol. 93, no. 11, pp. 1890-1908, Nov. 2005.

2. J. H. R. Enslin, "Renewable energy as an economic energy source for remote areas," Renew. Energy, vol. 1, no. 2, pp. 243-248, Jan. 1991.

3. D. K. Molzahn et al., "A Survey of Distributed Optimization and Control Algorithms for Electric Power
Systems," IEEE Trans. Smart Grid, vol. 8, no. 6, pp 2941-2962, Nov. 2017.

4. P. Panciatici et al., "Advanced optimization methods for power systems," in 2014 Power Systems Computation Conference, Wrocław, Poland, 2014, pp. 1-18.

5. R. Baños, F. Manzano-Agugliaro, F. G. Montoya, C. Gil, A. Alcayde, and J. Gómez, "Optimization methods applied to renewable and sustainable energy: A review," Renew. Sustain. Energy Rev., vol. 15, no. 4, pp. 17531766, May 2011.

6. B. Stott, O. Alsac, and A. J. Monticelli, "Security analysis and optimization," Proc. IEEE, vol. 75, no. 12, pp. 1623-1644, 1987.

7. Z. A. Khan, S. Ahmed, R. Nawaz, A. Mahmood, and S. Razzaq, "Optimization based individual and cooperative DSM in Smart Grids: A review," in 2015 Power Generation System and Renewable Energy Technologies (PGSRET), Islamabad, 2015, pp. 1-6.

8. A. Immanuel and D. C. Chengaiah, "A Comprehensive Literature Survey on Recent Methods of Optimal Power Flow," p. 12.

9. T. Khatib, A. Mohamed, and K. Sopian, "A review of photovoltaic systems size optimization techniques," Renew. Sustain. Energy Rev., vol. 22, pp. 454-465, Jun. 2013.

10. Y. Wang, P. Yemula, and A. Bose, "Decentralized Communication and Control Systems for Power System Operation,” IEEE Trans. Smart Grid, vol. 6, no. 2, pp. 885-893, Mar. 2015.

11. E. Dall'Anese, Hao Zhu, and G. B. Giannakis, "Distributed Optimal Power Flow for Smart Microgrids," IEEE Trans. Smart Grid, vol. 4, no. 3, pp. 1464-1475, Sep. 2013.

12. "Distributed Generation: Definitions, Benefits, Technologies \& Challenges," Int. J. Sci. Res. IJSR, vol. 5, no. 7, pp. 1941-1948, Jul. 2016.

13. A. Y. S. Lam, B. Zhang, and D. Tse, "Distributed Algorithms for Optimal Power Flow Problem," ArXiv11095229 Cs Math, Sep. 2011.

14. H. Terelius, U. Topcu, and R. M. Murray, "Decentralized Multi-Agent Optimization via Dual Decomposition," IFAC Proc. Vol., vol. 44, no. 1, pp. 11245-11251, Jan. 2011.

15. K. S. Pandya and S. K. Joshi, "A SURVEY OF OPTIMAL POWER FLOW METHODS," p. 10, 2005.

16. S. Sinha and S. S. Chandel, "Review of recent trends in optimization techniques for solar photovoltaic-wind based hybrid energy systems," Renew. Sustain. Energy Rev., vol. 50, pp. 755-769, Oct. 2015.

17. "Review on distribution optimization techniques for electric power system.docx.".

18. B. Stott, J. Jardim, and O. Alsac, "DC Power Flow Revisited," IEEE Trans. Power Syst., vol. 24, no. 3, pp. 1290-1300, Aug. 2009.

19. K. Turitsyn, P. Sulc, S. Backhaus, and M. Chertkov, "Options for Control of Reactive Power by Distributed Photovoltaic Generators," Proc. IEEE, vol. 99, no. 6, pp. 1063-1073, Jun. 2011

20. J. S. Vardakas, N. Zorba, and C. V. Verikoukis, "A Survey on Demand Response Programs in Smart Grids: Pricing Methods and Optimization Algorithms," IEEE Commun. Surv. Tutor., vol. 17, no. 1, pp. 152-178, 2015.

21. F. Nishimura, R. D. Tabors, M. D. Ilic, and J. R. LacalleMelero, "Benefit optimization of centralized and decentralized power systems in a multi-utility 
environment," IEEE Trans. Power Syst., vol. 8, no. 3, pp. 1180-1186, Aug. 1993.

22. A. Kargarian et al., "Toward Distributed/Decentralized DC Optimal Power Flow Implementation in Future Electric Power Systems," IEEE Trans. Smart Grid, vol. 9, no. 4, pp. 2574-2594, Jul. 2018.

23. S. Schuler, U. Münz, and F. Allgöwer, "Decentralized state feedback control for interconnected systems with application to power systems," J. Process Control, vol. 24, no. 2, pp. 379-388, Feb. 2014.

24. B. R. Vellaboyana and J. A. T. Taylor, "Optimal Decentralized Control of DC-Segmented Power Systems," IEEE Trans. Autom. Control, vol. 63, no. 10, pp. 3616-3622, Oct. 2018.

25. A. Kargarian Marvasti, Y. Fu, S. DorMohammadi, and M. Rais-Rohani, "Optimal Operation of Active Distribution Grids: A System of Systems Framework," IEEE Trans. Smart Grid, vol. 5, no. 3, pp. 1228-1237, May 2014.

26. H. L. Willis, H. Tram, M. V. Engel, and L. Finley, "Optimization applications to power distribution," IEEE Comput. Appl. Power, vol. 8, no. 4, pp. 12-17, Oct. 1995.

27. "Distributed optimization approaches for emerging power systems operation_ A review _ Elsevier Enhanced Reader.html.".

28. "A composable method for real-time control of active distribution networks with explicit power setpoints. Part I_Framework Elsevier Enhanced Reader.html."

29. "Applied Mathematics for Restructured Electric Power Systems_Optimization ... - Google Books.html.".

30. W. Qiu, V. Vittal, and M. Khammash, "Decentralized Power System Stabilizer Design Using Linear Parameter Varying Approach," IEEE Trans. Power Syst., vol. 19, no. 4, pp. 1951-1960, Nov. 2004.

31. A. G. Bakirtzis and P. N. Biskas, "A decentralized solution to the DC-OPF of interconnected power systems," IEEE Trans. Power Syst., vol. 18, no. 3, pp. 1007-1013, Aug. 2003.

32. H. J. Liu, W. Shi, and H. Zhu, "Dynamic decentralized voltage control for power distribution networks," in 2016 IEEE Statistical Signal Processing Workshop (SSP), Palma de Mallorca, Spain, 2016, pp. 1-5.

33. [33] G. Cohen, "Optimization by decomposition and coordination: A unified approach," IEEE Trans. Autom. Control, vol. 23, no. 2, pp. 222-232, Apr. 1978.

34. T. Erseghe, "Distributed Optimal Power Flow Using ADMM," IEEE Trans. Power Syst., vol. 29, no. 5, pp. 2370-2380, Sep. 2014.

35. P. Sulc, S. Backhaus, and M. Chertkov, "Optimal Distributed Control of Reactive Power Via the Alternating Direction Method of Multipliers," IEEE Trans. Energy Convers., vol. 29, no. 4, pp. 968-977, Dec. 2014. 